\title{
RS CVn binary IM Peg - investigation of stellar activity and surface flows
}

\author{
Heidi Korhonen ${ }^{1}$, Michael Weber ${ }^{2}$, Markus Wittkowski ${ }^{1}$, \\ Thomas Granzer ${ }^{2}$ and Klaus G. Strassmeier ${ }^{2}$ \\ ${ }^{1}$ European Southern Observatory, Karl-Schwarzschild-Str. 2, D-85748 Garching bei München, \\ Germany \\ ${ }^{2}$ Astrophysical Institute Potsdam, An der Sternwarte 16, D-14482 Potsdam, Germany
}

\begin{abstract}
We have obtained high resolution, high S/N spectra of the RS CVn binary IM Peg using UVES spectrograph at Kueyen $8.2 \mathrm{~m}$ telescope of ESO VLT. We use Doppler imaging techniques to obtain stellar surface temperature maps from the UVES data. The TempMap code allows us to use surface differential rotation as an input parameter and thus to try to construct the rotation pattern on the stellar surface as part of the inversion process. The UVES observations are combined with spectroscopic observations from another time period obtained at the STELLA observatory. We obtain stellar surface temperature maps also from these spectra. These Doppler images are used to study the magnetic activity and surface differential rotation on IM Peg.
\end{abstract}

Keywords. stars: activity, chromospheres, spots

\section{Target and observations}

IM Peg is an active RS CVn binary with an orbital period of 24.45 days. The secondary showing signs for both photospheric and chromospheric activity is of spectral type K2III, and has rotational velocity of $\operatorname{vsin} i=28 \mathrm{~km} / \mathrm{s}$. IM Peg is a quite well studied system of which several surface temperature maps showing spots at very high latitudes have been obtained (e.g., Berdyugina et al. 2000).

Very high $\mathrm{S} / \mathrm{N}$ and high resolution spectroscopic observations were obtained at the European Southern Observatory with UVES (Ultraviolet and Visual Echelle Spectrograph) mounted on the 8-m Kueyen telescope of the VLT. The observations obtained in October-December 2008 have a spectral resolution of 110000 and S/N of 350 .

More high resolution spectra were obtained between June 26 and July 17 2009, at the fully robotic 1.2-m STELLA observatory in Tenerife. The fibre-fed SES spectrograph gives a resolving power of 55000 and a wavelength coverage of 3900-9000 A. The typical $\mathrm{S} / \mathrm{N}$ of the observations was 250 at the peak, but approximately $50 \%$ less in the spectra region used for Doppler imaging.

\section{Spots and surface flows}

The Doppler images were obtained using the TempMap inversions code (Rice et al. 1989). The observations are compared to a grid of local line profiles calculated using SPECTRUM spectral synthesis code (Gray \& Corbally 1994) and Kurucz model atmospheres (Kurucz 1993). Before inversions the STELLA data were de-noised using 15 of 401 components of a PCA analysis using 747 spectral lines (Carroll et al. 2007). In the inversion results shown here only the Fe I $6430 \AA$ line has been used. 

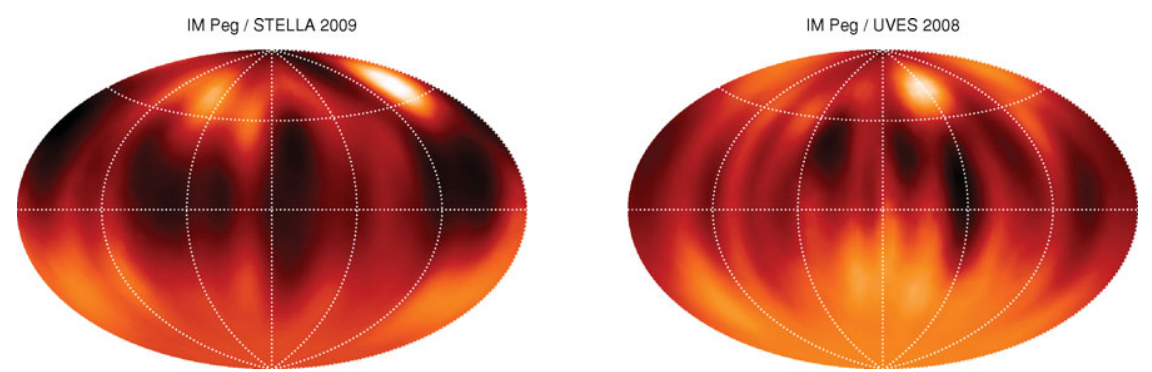

Figure 1. Temperature maps obtained from the UVES and STELLA data using Doppler imaging techniques and Fe I $6430 \AA$ line.

The surface flows on stars can be investigated from Doppler images. In one commonly used method two temperature maps close in time are cross-correlated to study the shift of the surface features between the maps. Another way is to introduce the flow pattern in the Doppler imaging code and look for the parameter set that minimises the deviation between the observations and the model (so-called $\chi^{2}$-landscape). Fig. 2 shows the $\chi^{2}$ landscape for the UVES dataset of IM Peg. The result shows quite a shallow minimum indicating anti-solar surface differential rotation, i.e., with the pole rotating faster than the equator. This result is similar to the one presented by Weber (2007).

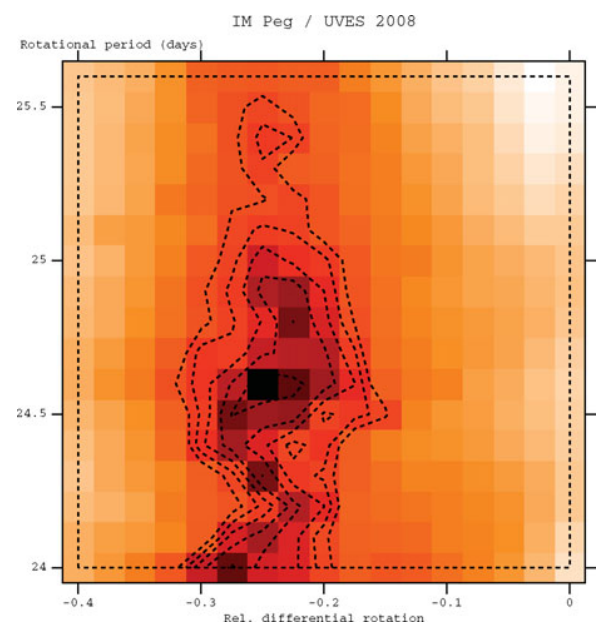

Figure 2. The goodness of fit parameter for the UVES dataset with varying period and differential rotation.

\section{Chromospheric activity}

The $\mathrm{H} \alpha$ profiles from all the epochs obtained with UVES are plotted in Fig. 3. In IM Peg the $\mathrm{H} \alpha$ line is in absorption, and shows clear variations with time. For studying in detail the activity in the upper atmosphere a dynamic spectrum, also shown in Fig. 3, was obtained from the $\mathrm{H} \alpha$ profiles. For showing the variations better an average spectrum (shown as a thick line in the profile plot) was first removed from all the profiles. The dynamical spectrum shows enhanced emission around phase $1.0(0.0)$, most likely from plages. Signs of a flare are seen in the phase 0.98 . The extra absorption at the phase 0.43 gives some indication of cool chromospheric clouds. 

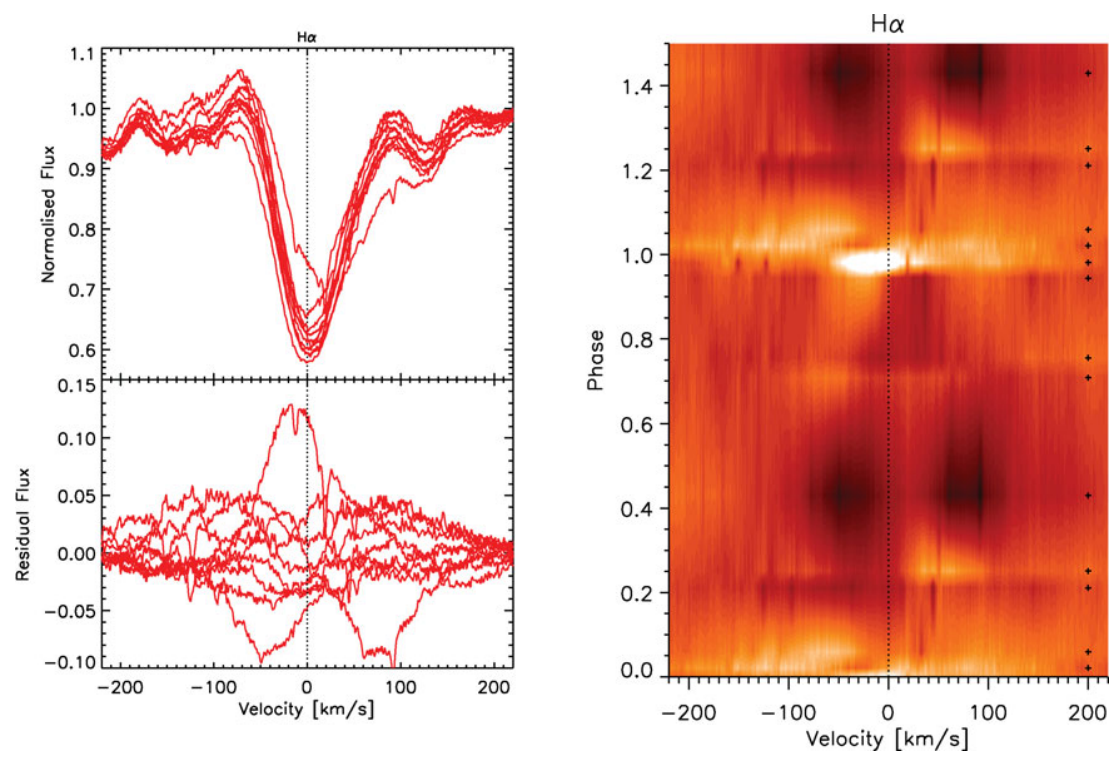

Figure 3. Left: All the $\mathrm{H} \alpha$ profiles from the UVES data. Upper plot shows the profiles normalised to the continuum, and the lower plot the residual profiles after subtracting the mean profile (thick line in the upper plot). Right: Dynamic spectrum of the $\mathrm{H} \alpha$ line based on the residual profiles. The $\mathrm{x}$-axis gives the velocity and the $\mathrm{y}$-axis the phase. The crosses on the right hand side of the plot give the phases of the observations and the dotted line the zero velocity. Brighter the colour the more emission is observed. The phases go from 0.0 to 1.5 to better show the flare occurring near the phase $0.0(1.0)$.

\section{References}

Berdyugina, S. V., Berdyugin, A. V., Ilyin, I., \& Tuominen, I. 2000, A\& A, 360, 272

Carroll, T. A., Kopf, M., Ilyin, I., \& Strassmeier, K. G., 2007 AN, 328, 1043

Gray, R. O. \& Corbally, C. J. 1994, AJ, 107, 742

Kurucz, R. L. 1993, Kurucz CD No. 13

Rice, J. B., Wehlau, W. H., \& Khokhlova, V. L. 1989, A\& A, 208, 179

Strassmeier, K. G., Granzer, Th., Weber, M., et al. 2004, AN, 325, 527

Weber, M., Granzer, Th., Strassmeier, K. G., \& Woche, M. 2008, SPIE, 7019, 70190L

Weber, M. $2007 A N, 328,1075$ 